\title{
On the Distribution of Type II Errors in Hypothesis Testing
}

\author{
Skip Thompson \\ Department of Mathematics and Statistics, Radford University Radford, USA \\ E-mail: thompson@radford.edu \\ Received October 16, 2010; revised November 26, 2010; accepted November 30, 2010
}

\begin{abstract}
When a statistical test of hypothesis for a population mean is performed, we are faced with the possibility of committing a Type II error by not rejecting the null hypothesis when in fact the population mean has changed. We consider this issue and quantify matters in a manner that differs a bit from what is commonly done. In particular, we define the probability distribution function for Type II errors. We then explore some interesting properties that we have not seen mentioned elsewhere for this probability distribution function. Finally, we discuss several Maple procedures that can be used to perform various calculations using the distribution.
\end{abstract}

Keywords: Complementary Error Function, Hypothesis Testing, Power Curves, Power Surfaces, Type II Errors

\section{Introduction}

Both the probability $\alpha$ of committing a Type I error and the probability $\beta$ of committing a Type II error must be considered when a statistical test of hypothesis of a population mean is performed. There is a vast literature dealing with the role of each type of error. Both [1] and [2] contain useful discussions and references to the relevant literature. For a given sample size, it is possible to calculate and control $\alpha$ directly; but it is not possible to calculate $\beta$ since the new population mean is not known. Various techniques have been developed to quantify the role of Type II errors. A particularly good description of these techniques may be found in [1]. For example, operating-characteristic curves are often used to estimate sample sizes needed to keep the probability of a Type II error below a prescribed level. Similarly, the power of a test is used to assess the ability of a test to detect changes in the population mean. For a given sample size, it is customary to postulate a new value (or several new values) for the population mean and compute $\beta$ using each such mean. The size of $\beta$ then gives an indication whether the sample size is adequate.

In this paper we will maintain the spirit of this approach but we will quantify Type II errors using a different perspective. In Section 2, we will briefly review Type II errors. We will use $\beta(u)$ to denote the proba- bility of a Type II error if the new population mean is equal to $u$. In Section 3, we will go a bit further and convert $\beta(u)$ into a probability distribution $\beta_{u}(u)$ and explore properties of this distribution. In Section 4, we will illustrate how the distribution can be used to answer interesting questions that are usually addressed using operating curves and power curves and how it may be used to quantify conventional wisdom regarding Type II errors. By converting $\beta(u)$ into a probability distribution, we will find that these questions can be addressed in a systematic and convenient manner.

\section{Type II Errors}

In this section we review Type II errors briefly. A detailed discussion of Type II errors (and hypothesis testing in general) can be found in any mathematical statistics text, for example, [2]. We assume that the parent population of interest is normally distributed with standard deviation $\sigma$. If $\alpha$ is the significance level for a two tailed test, the null hypothesis $u=u_{0}$ will not be rejected for a sample of size $n$ if the sample mean $\bar{x}$ is such that the standardized statistic

$$
z=\frac{\bar{x}-u_{0}}{\sigma / \sqrt{n}}
$$

falls in the interval 


$$
\left(u_{0}-L\left(\frac{\sigma}{\sqrt{n}}\right), u_{0}+L\left(\frac{\sigma}{\sqrt{n}}\right)\right)
$$

where $L$ denotes the inverse standard normal value determined by the right tail of size $\frac{\alpha}{2}$.

We will use the complementary error function to facilitate our discussion. This function is defined as

$$
\operatorname{erfc}(x)=1-\frac{2}{\sqrt{\pi}} \int_{0}^{x} e^{-t^{2}} d t
$$

We note the following useful properties of the complementary error function.

$$
\begin{gathered}
\lim _{x \rightarrow \infty} \operatorname{erfc}(x)=0 \\
\lim _{x \rightarrow-\infty} \operatorname{erfc}(x)=2 \\
\int \operatorname{erfc}(x) d x=x \operatorname{erfc}(x)-\frac{e^{-x^{2}}}{\sqrt{\pi}}
\end{gathered}
$$

The cumulative distribution function for the standard normal distribution can be expressed using the complementary error function as

$$
\frac{1}{\sqrt{2 \pi}} \int_{-\infty}^{x} e^{-t^{2} / 2} d t=\frac{1}{2} \operatorname{erfc}(-x / \sqrt{2})
$$

The probability of a Type II error is equal to

$$
\beta(u)=\frac{1}{\sqrt{2 \pi}} \int_{M_{2}(u)}^{M_{1}(u)} e^{-t^{2} / 2} d t
$$

where $M_{1}$ and $M_{2}$ are the $u$-based standardized values defined by

$$
M_{2,1}(u)=\frac{u_{0} \pm L \sigma / \sqrt{n}-u}{\sigma / \sqrt{n}}
$$

so that

$$
\beta(u)=\left.\frac{1}{2} \operatorname{erfc}(-t / \sqrt{2})\right|_{M_{1}(u)} ^{M_{2}(u)}
$$

The probability of a Type II error approaches a maximum limiting value $1-\alpha$ as $u \rightarrow u_{0}$. Furthermore, a bit of reflection shows that $\beta(u)$ is symmetric about $u_{0}$. We define a probability distribution for $\beta(u)$ as follows. Let

$$
T_{\beta}=\left.\frac{1}{2} \int_{-\infty}^{\infty} \operatorname{erfc}(-t / \sqrt{2})\right|_{M_{1}(u)} ^{M_{2}(u)} d u
$$

The probability distribution is then

$$
\beta_{u}(u)=\frac{\beta(u)}{T_{\beta}}
$$

Of course, in any practical tests of hypothesis, the new population mean $u$ is not a random variable. We are being cavalier and regarding it as such simply for the purposes of analyzing the properties of $\beta_{u}(u)$. Quantities obtained by integrating $\beta_{u}(u)$ can be interpreted simply as the fraction of all possible new population means that yield Type II errors of various sizes.

\section{Distribution Properties of Type II Errors}

In this section we will explore several important and interesting properties of the $\beta_{u}(u)$ distribution.

Property 1. We claim that $T_{\beta}$, interestingly enough, is equal to the length of the $1-\alpha$ confidence interval about $u_{0}$, that is,

$$
T_{\beta}=2 L\left(\frac{\sigma}{\sqrt{n}}\right)
$$

When the integral in Equation (11) is expanded, there results an expression with fifteen terms. (Refer to [3] for the actual expression and simplification.) Due to Equations (2)-(4), all but two eight terms approach 0 as $u_{2} \rightarrow \infty$ and $u_{1} \rightarrow-\infty$ since $M_{1}(u)$ and $M_{2}(u)$ approach $\pm \infty$ as $u \rightarrow \mp \infty$. The remaining two nonzero terms are

$$
\frac{\sigma}{\sqrt{2 n}}\left(\frac{u_{0}+\frac{L \sigma}{\sqrt{n}}-u_{1}}{\frac{\sqrt{2} \sigma}{\sqrt{n}}} \operatorname{erfc}\left(-\frac{u_{0}+\frac{L \sigma}{\sqrt{n}}-u_{1}}{\frac{\sqrt{2} \sigma}{\sqrt{n}}}\right)\right)
$$

and

$$
-\frac{\sigma}{\sqrt{2 n}}\left(\frac{u_{0}-\frac{L \sigma}{\sqrt{n}}-u_{1}}{\frac{\sqrt{2} \sigma}{\sqrt{n}}} \operatorname{erfc}\left(-\frac{u_{0}-\frac{L \sigma}{\sqrt{n}}-u_{1}}{\frac{\sqrt{2} \sigma}{\sqrt{n}}}\right)\right)
$$

The arguments in the erfc factors approach $-\infty$ as $u_{1} \rightarrow-\infty$; so each factor approaches 2 . Therefore,

$$
\begin{aligned}
T_{\beta} & =\frac{2 \sigma}{\sqrt{2 n}}\left(\frac{u_{0}+L \sigma / \sqrt{n}-u_{1}}{\sqrt{2} \sigma / \sqrt{n}}-\frac{u_{0}-L \sigma / \sqrt{n}-u_{1}}{\sqrt{2} \sigma / \sqrt{n}}\right) \\
& =2 L\left(\frac{\sigma}{\sqrt{n}}\right)
\end{aligned}
$$

as claimed. As a matter of interest, we give also a more conventional proof (based on the standard normal rather than the complementary error function) of the fact that

$$
\int_{-\infty}^{\infty} \beta(u) d u=2 L\left(\frac{\sigma}{\sqrt{n}}\right) .
$$

Indeed, 


$$
\begin{aligned}
& \int_{-\infty}^{\infty} \beta(u) d u=\frac{1}{\sqrt{2 \pi}} \int_{-\infty}^{\infty} \int_{\frac{u_{0}-u}{\sigma / \sqrt{n}}-L}^{\frac{u_{0}-u}{\sigma / \sqrt{n}}+L} e^{-x^{2} / 2} d x d u \\
& =\frac{1}{\sqrt{2 \pi}}\left(\frac{\sigma}{\sqrt{n}}\right) \int_{-\infty}^{\infty} \int_{w-L}^{w+L} e^{-x^{2} / 2} d x d w \\
& =\frac{1}{\sqrt{2 \pi}}\left(\frac{\sigma}{\sqrt{n}}\right) \int_{-\infty}^{\infty} \int_{w-L}^{w+L} e^{-x^{2} / 2} d w d x \\
& =\frac{1}{\sqrt{2 \pi}}\left(\frac{\sigma}{\sqrt{n}}\right) \int_{-\infty}^{\infty}\left((x+L) e^{-x^{2} / 2}-(x-L) e^{-x^{2} / 2}\right) d x \\
& =\frac{1}{\sqrt{2 \pi}}\left(\frac{\sigma}{\sqrt{n}}\right)(2 L) \int_{-\infty}^{\infty} e^{-x^{2} / 2} d x \\
& =\frac{1}{\sqrt{2 \pi}}\left(\frac{\sigma}{\sqrt{n}}\right)(2 L)(\sqrt{2 \pi})=2 L\left(\frac{\sigma}{\sqrt{n}}\right)
\end{aligned}
$$

Property 2. Given values $u_{1}$ and $u_{2}$, we have

$$
p\left(u_{1} \leq u \leq u_{2}\right)=\left.\frac{1}{2 T_{\beta}} \int_{u_{1}}^{u_{2}} \operatorname{erfc}(-t / \sqrt{2})\right|_{M_{1}(u)} ^{M_{2}(u)} d u
$$

so that

$$
\begin{aligned}
& p\left(u_{1} \leq u \leq u_{2}\right) \\
& =\frac{1}{2 T_{\beta}} \int_{u_{1}}^{u_{2}}\left(\operatorname{erfc}\left(-\frac{M_{2}(u)}{\sqrt{2}}\right)-\operatorname{erfc}\left(-\frac{M_{1}(u)}{\sqrt{2}}\right)\right) d u
\end{aligned}
$$

which in turn is equal to

$$
\frac{1}{2 T_{\beta}} \int_{u_{1}}^{u_{2}}\left(\operatorname{erfc}\left(-\frac{u_{0}+\frac{L \sigma}{\sqrt{n}}-u}{\frac{\sqrt{2} \sigma}{\sqrt{n}}}\right)-\operatorname{erfc}\left(-\frac{u_{0}-\frac{L \sigma}{\sqrt{n}}-u}{\frac{\sqrt{2} \sigma}{\sqrt{n}}}\right)\right) d u
$$

Breaking this integral into two, using the substitutions $x=-\frac{M_{i}(u)}{\sqrt{2}}$, and using Equation (4), we see that

$$
\begin{aligned}
p\left(u_{1} \leq u \leq u_{2}\right)= & \frac{\sigma}{\sqrt{2 n} T_{\beta}}\left[\left.\left(x \operatorname{erfc}(x)-\frac{e^{-x^{2}}}{\sqrt{\pi}}\right)\right|_{-\frac{M_{2}\left(u_{1}\right)}{\sqrt{2}}} ^{-\frac{M_{2}\left(u_{2}\right)}{\sqrt{2}}}\right. \\
& \left.-\left.\left(x \operatorname{erfc}(x)-\frac{e^{-x^{2}}}{\sqrt{\pi}}\right)\right|_{-M_{1}\left(u_{1}\right) / \sqrt{2}} ^{-M_{1}\left(u_{2}\right) / \sqrt{2}}\right]
\end{aligned}
$$

Equation (12) allows us to work with the probability distribution $\beta_{u}(u)$ using the erfc function without the need to integrate it directly.

Property 3. If we use Equation (3) and Property 1, and we let $u_{1} \rightarrow-\infty$, we find that the contribution of the two terms $M_{i}\left(u_{1}\right)$ is 1 . We thus obtain a convenient representation for the cumulative distribution function for $\beta_{u}(u)$

$$
\begin{aligned}
& p(-\infty<x \leq u) \\
& =1-\frac{\sigma}{\sqrt{2 n} T_{\beta}}\left(\left.\left(x \operatorname{erfc}(x)-\frac{e^{-x^{2}}}{\sqrt{\pi}}\right)\right|_{-M_{2}(u) / \sqrt{2}} ^{-M_{1}(u) / \sqrt{2}}\right)
\end{aligned}
$$

Property 4. For a given value of $p$ in $(0,1-\alpha]$, denote by $u_{L}$ and $u_{R}$ the values of $u$ for which $\beta(u)=p$ with $u_{L}<u_{R}$. (We refer to these values as the left and right inverses of $p$, respectively.) In this case, Equation (13) can be expressed in a simpler form that more clearly shows the dependence on $p$ and $L$ :

$$
\begin{aligned}
p\left(u_{1} \leq u \leq u_{2}\right) & =1-\frac{1}{2} \operatorname{erfc}\left(-\frac{M_{1}\left(u_{L}\right)}{\sqrt{2}}\right)-\frac{p}{L} M_{2}\left(u_{L}\right) \\
& -\frac{1}{\sqrt{2 \pi} L}\left(e^{-M_{2}^{2}\left(u_{L}\right) / 2}-e^{-M_{1}^{2}\left(u_{L}\right) / 2}\right)
\end{aligned}
$$

Indeed, using Equation 13 shows that $p\left(-\infty<x \leq u_{L}\right)$ is equal to

$$
1-\frac{\sigma}{2 \sqrt{2 n} T_{\beta}}\left(\left.\left(x \operatorname{erfc}(x)-\frac{e^{-x^{2}}}{\sqrt{\pi}}\right)\right|_{-M_{2}\left(u_{L}\right) / \sqrt{2}} ^{-M_{1}\left(u_{L}\right) / \sqrt{2}}\right)
$$

Expanding this expression using Property 4 and Equation (11) yields

$$
\begin{aligned}
& 1-\frac{1}{2 \sqrt{2} L}\left(\frac{M_{2}\left(u_{L}\right)}{\sqrt{2}} \operatorname{erfc}\left(\frac{-M_{2}\left(u_{L}\right)}{\sqrt{2}}\right)\right. \\
& \left.-\frac{M_{1}\left(u_{L}\right)}{\sqrt{2}} \operatorname{erfc}\left(\frac{-M_{1}\left(u_{L}\right)}{\sqrt{2}}\right)\right) \\
& -\frac{1}{2 \sqrt{2 \pi} L}\left(e^{-M_{2}^{2}\left(u_{L}\right) / 2}-e^{-M_{1}^{2}\left(u_{L}\right) / 2}\right)
\end{aligned}
$$

We can rewrite the factor containing the two values of erfc as

$$
\begin{aligned}
& \frac{M_{2}\left(u_{L}\right)}{\sqrt{2}}\left(\operatorname{erfc}\left(\frac{-M_{2}\left(u_{L}\right)}{\sqrt{2}}\right)-\operatorname{erfc}\left(\frac{-M_{1}\left(u_{L}\right)}{\sqrt{2}}\right)\right) \\
& +\operatorname{erfc}\left(\frac{-M_{1}\left(u_{L}\right)}{\sqrt{2}}\right)\left(\frac{M_{2}\left(u_{L}\right)}{\sqrt{2}}-\frac{M_{1}\left(u_{L}\right)}{\sqrt{2}}\right)
\end{aligned}
$$

Since $\beta\left(u_{L}\right)=p$, the first parenthesized term is equal to $2 p$. The second parenthesized term is equal to $\sqrt{2} L$. Making these substitutions and simplifying establishes Equation (14).

Property 5. The mean of this distribution is equal to $u_{0}$ due to symmetry. The standard distribution is equal to

$$
\frac{\sigma}{\sqrt{n}} \sqrt{1+L^{2} / 3}
$$


For values of $\alpha$ in the range 0.01 to $0.10, \sqrt{1+L^{2} / 3}$ ranges from approximately 1.5 to 2 . The size of this factor accounts in part for the rounded shape of $\beta_{u}(u)$. To establish this property, we start with the integral

$$
\frac{2}{T_{\beta}} \int_{-\infty}^{u_{0}}\left(u-u_{0}\right)^{2} \beta_{u}(u) d u
$$

We obtain a complicated antiderivative with twenty-six terms. (Refer to [3] for the actual expression and simplification.) However, grouping terms and using Equations (2) and (3) show that all but two of the terms approach 0 as $u_{1} \rightarrow-\infty$. The two grouped terms that do not approach 0 as $u_{1} \rightarrow-\infty$ are

$$
\frac{1}{2 L s} \lim _{u_{1} \rightarrow-\infty} L s^{3}\left(\operatorname{erfc}\left(\frac{-u_{1}-u_{0}+L s}{\sqrt{2} s}\right)-\operatorname{erfc}\left(\frac{u_{1}+u_{0}+L s}{\sqrt{2} s}\right)\right)
$$

and

$\frac{1}{6 L s} \lim _{u_{1} \rightarrow-\infty} L^{3} s^{3}\left(\operatorname{erfc}\left(\frac{-u_{1}-u_{0}+L s}{\sqrt{2} s}\right)-\operatorname{erfc}\left(\frac{u_{1}+u_{0}+L s}{\sqrt{2} s}\right)\right)$

In both grouped terms

$$
\operatorname{erfc}\left(\frac{-u_{1}-u_{0}+L s}{\sqrt{2} s}\right) \rightarrow 0 \operatorname{asu}_{1} \rightarrow-\infty
$$

and

$$
\operatorname{erfc}\left(\frac{u_{1}+u_{0}+L s}{\sqrt{2} s}\right) \rightarrow 2 \mathrm{as} u_{1} \rightarrow-\infty
$$

Making these substitutions and simplifying leads to

$$
\frac{2}{T_{\beta}} \int_{-\infty}^{u_{0}}\left(u-u_{0}\right)^{2} \beta_{u}(u) d u=s^{2}\left(1+L^{2} / 3\right)
$$

as claimed.

Property 6. Working with the second derivative of $\beta_{u}(u)$ shows that the inflection points of $\beta_{u}(u)$ occur when

$$
u=u_{0} \pm\left(\frac{s}{2 L}\right) t_{*}
$$

where $t_{*}$ is the unique positive solution of

$$
-t e^{t}+t+2 L^{2}+2 L^{2} e^{t}=0 .
$$

(Refer to [3] for details.) We note that $t_{*}$ is in the interval $\left(2 L^{2}, 3 L^{2}\right)$ for $L>\sqrt{\ln (5) / 3}$.

Property 7. Given an interval $\left[u_{1}, u_{2}\right]$ that we suspect contains the new population mean, the average probability of a Type II error for this interval is equal to

$$
\frac{T_{\beta}}{\left(u_{2}-u_{1}\right)} p\left(u_{1} \leq u \leq u_{2}\right)
$$

Customarily, $\beta(u)$ is calculated for a particular value of the population mean or for a few particular values.
This simple property provides an interval-oriented version of $\beta(u)$. By dropping the factor of $T_{\beta}$, we can obtain similar average values for $\beta_{u}(u)$.

Property 8. Given a probability level $p$, the probability that $\beta(u)$ does not exceed $p$ is equal to

$$
p_{*}(p)=2 \int_{-\infty}^{u_{L}(p)} \beta_{u}(u) d u
$$

where $u_{L}(p)$ is the left inverse of $p=\beta\left(u_{L}(p)\right)$. To see this, first note that we can calculate $p_{*}(p)$ using Property 4. Suppose $p_{*}$ is constructed using two sets of population parameters $u_{0}, \sigma_{0}$, and $n_{0}$, and $u_{1}, \sigma_{1}$, and $n_{1}$. The definition of $M_{1}\left(u_{L}\right)$ and $M_{2}\left(u_{L}\right)$ and the fact that $\beta_{0}\left(u_{L_{0}}\right)=\beta_{1}\left(u_{L_{1}}\right)=p$ leads to

$$
\frac{u_{0}-u_{L_{0}}}{\frac{\sigma_{0}}{\sqrt{n_{0}}}}=\frac{u_{1}-u_{L_{1}}}{\frac{\sigma_{1}}{\sqrt{n_{1}}}}
$$

Solving for $u_{L_{1}}$ in terms of $u_{L_{0}}$ and substituting the results into Property 4 for the second set of parameters shows that the corresponding terms in Property 4 are equal for the two sets of parameters so that $p_{*, 0}(p)=$ $p_{*, 1}(p) . p_{*}$ is thus a function of $p$ and $\alpha$ (via $L$ ). $p_{*}$ quantifies intrinsically the well-known difficulty of obtaining Type II errors within prescribed levels due to the roundedness of $\beta(u)$.

Property 9. A slight extension of Property 8 is possible. Given two probability levels $p_{1}$ and $p_{2}$ with $p_{1}<p_{2}$, the probability that $\beta(u)$ will be between these values is equal to

$2\left[\operatorname{beta} \_c d f\left(u_{L, 2}\right)-\right.$ beta_cdf $\left.\left(u_{L, 1}\right)-\frac{p_{1}}{T_{\beta}}\left(u_{L, 2}-u_{L, 1}\right)\right]$

where $u_{L, 1}$ and $u_{L, 2}$ are the left inverses of $p_{1}$ and $p_{2}$, respectively.

\section{Using $\beta(u)$ and $\beta_{u}(u)$}

The Maple Computer Algebra System [4] can be used to illustrate various calculations required to address questions of interest. Relevant calculations are implemented in a Maple worksheet [3] and several auxiliary worksheets that are available from the author's web site. In the procedures discussed here, beta_erfc is the function defined by Equation 8 and beta_cdf is the cumulative probability distribution function defined by Equation (13). fsolve is the Maple nonlinear equation solver. It should be noted that the actual procedures in [3] are a bit more complicated due to the need for error checking and the need to deal with numerical difficulties caused by the effects of floating point calculations; but we won't fuss about the details here. Interested readers may wish to consider implementing similar procedures using their fa- 
vorite statistical computing package.

The uses of $\beta(u)$ are well known [2]. For example, given a particular value $u_{1}$ for the new population mean we can calculate the probability $\beta\left(u_{1}\right)$ of a Type II error using Equation (8) or we can perform the calculation as usual using Equation (6). Furthermore, given an interval $\left[u_{1}, u_{2}\right]$ that we suspect contains the new population mean, we can calculate the average probability of a Type II error for this interval using Property 7.

Power curves and operating-characteristic curves [1] are often used to help determine appropriate sample sizes to obtain Type II error probabilities of different sizes. Such a curve is the graph of $1-\beta$ obtained using various sample sizes. Rather than generate a set of one-dimensional operating-characteristic curves in the usual fashion we can consider $\beta(u)$ as a function of $u$ and $n$ and plot the surface $1-\beta(u, n)$ or the surface the $\beta(u, n)$ as in the following abbreviated code segment.

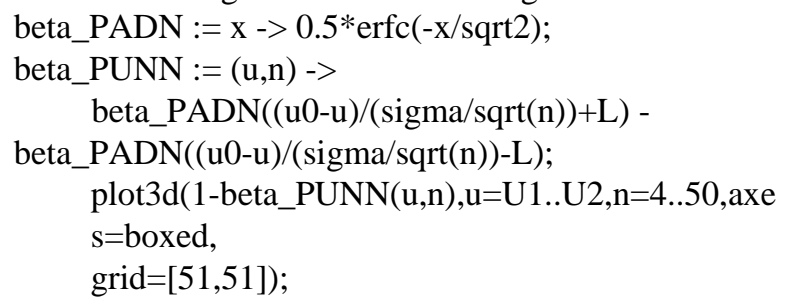

The surface can be rendered in various ways. Figure 1 depicts a power surface for a typical set of population parameters. By working with the surface contours and cross sectional slices, we can obtain the information usually obtained by using one-dimensional power curves. In particular, we can study the question of determining the sample sizes required to yield Type II errors of various sizes. To see how we might proceed, consider the following example. Suppose the population parameters are $u_{0}=$ 74 and $\sigma=30$. Further suppose we wish to use a significance level $\alpha=0.01$. We would like to determine the minimum sample size that yields a Type II error equal to 0.2 when the new population mean is equal to 85 . While it is simple enough to solve the nonlinear equation $\beta\left(u, n_{\min }\right)=0.02$, we can use the power surface to estimate $n_{\min }$ as accurately as desired. Figure 2 shows the portion of the surface for which $\beta(u, n) \geq 0.2$. If we follow the surface around the bottom for $u=85$ until reaching the contour curve for $u=85$, we see that a sample size between 85 and 90 will suffice. Solving the corresponding nonlinear equation shows that $n_{\min }=87$. For this example, $n_{\min }=87$ agrees with the usual two-tailed estimate [2] and this approach is applicable to other types of tests in which a simple estimate is not readily available. The usefulness of this approach is enhanced due to the fact the $\beta(u)$ surface can be generated quickly without the need to perform tedious and time consuming integrations. Also, once the surface has been generated, it

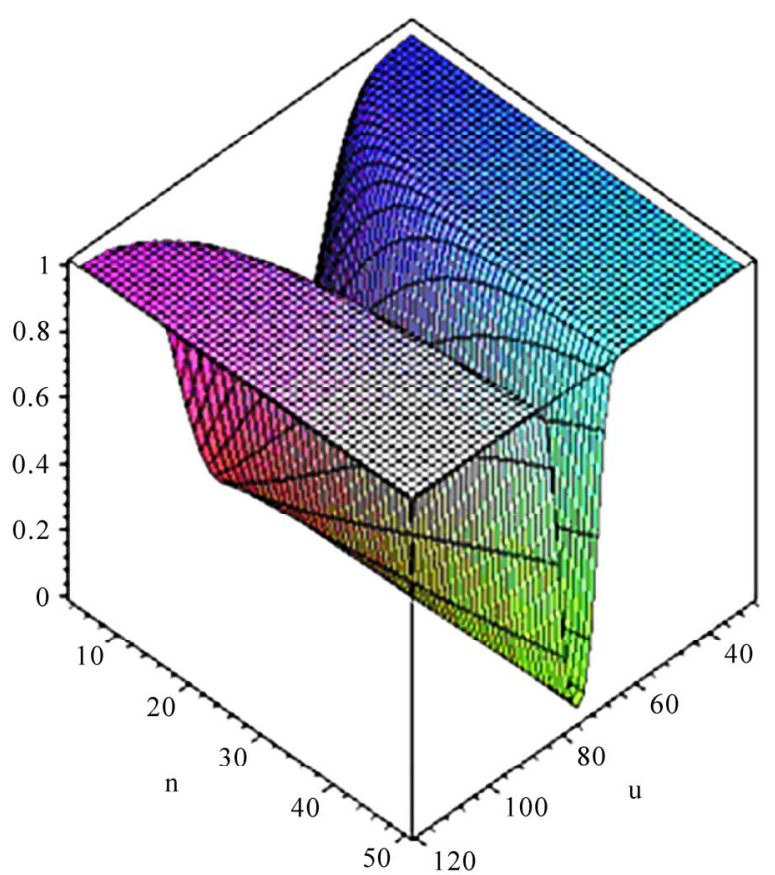

Figure 1. The power surface $1-\beta(u, n)$.

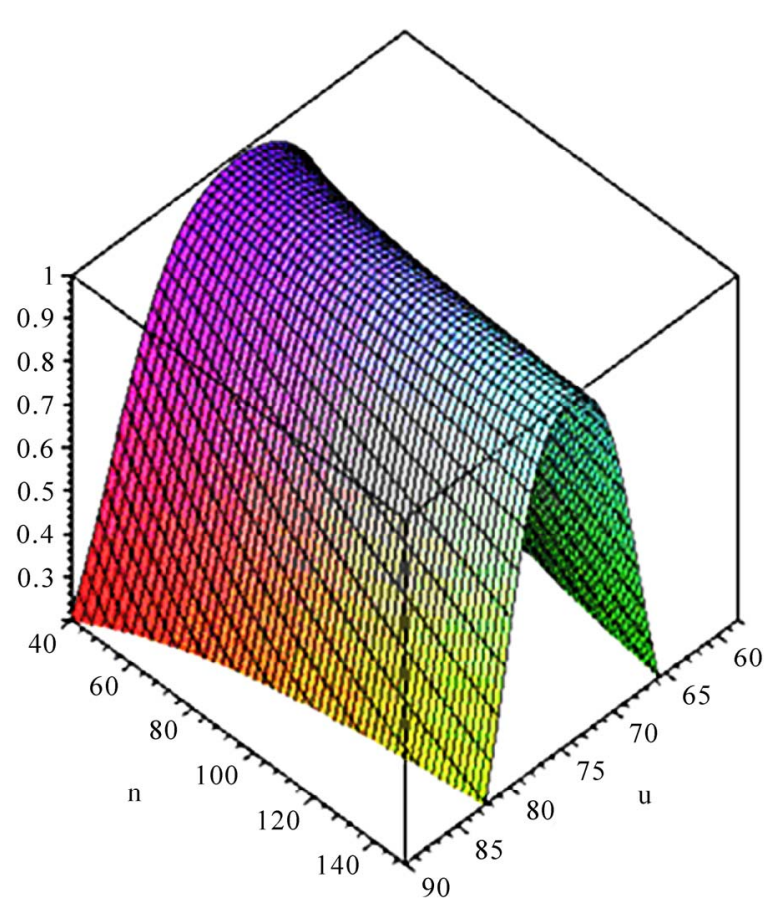

Figure 2. Top portion of the surface $\beta(u, n)$.

can viewed and manipulated in any manner that is desired.

Similarly, by considering $\beta(u)$ as a function of $u$ and $\alpha$, we can plot the power surface $1-\beta(u, \alpha)$ as in the following abbreviated code segment. 


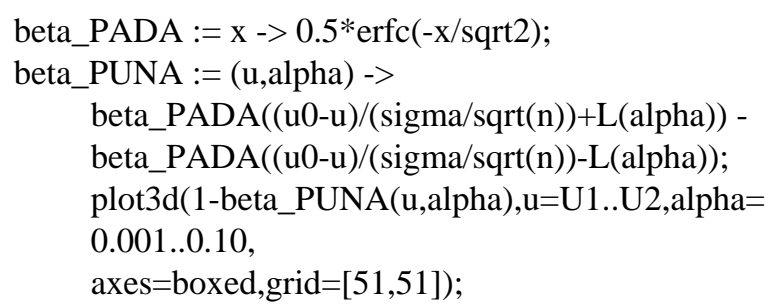

Figure 3 depicts the surface obtained for a typical set of population parameters. As with Figure 1 we can use the surface contours and cross sectional slices to consider the effect of using different significance levels.

For the same reason that inverse normal calculations are needed when working with a normal distribution, we need to be able to invert $\beta_{u}(u)$ to find the value of $u_{p}$ for which $p\left(x \leq u_{p}\right)=p$. The following procedure can be used to perform this task. It uses fsolve to find $u$ given that $p=$ beta_cdf $(u)$.

beta_cdf_inv $:=\operatorname{proc}(\mathrm{p})$

\# Calculate the inverse of beta_cdf.

local eqn, u, up:

global beta_cdf, U1, U2, u0:

u := 'u': up := 'up':

if $(p=0.5)$ then

$\mathrm{u}:=\mathrm{u} 0$ :

return(u);

fi:

if $(\mathrm{p}<0.5)$ then
eqn $:=$ beta_cdf(up) $=\mathrm{p}$ :

fsolve(eqn, $\{$ up $\}$, U1..u0):

assign(\%):

else

eqn $:=$ beta_cdf(up) $=\mathrm{p}$ :

fsolve(eqn, \{up\}, u0..U2):

fi:

assign(\%):

u := up:

return(u):

end proc:

Given a particular probability $p$, we can perform an inverse calculation to find the values $u_{L}<u_{0}$ and $u_{R}>u_{0}$ for which $\beta\left(u_{L}\right)=\beta\left(u_{R}\right)=p$ in much the same way as in the above inversion of $\beta_{u}(u) . u_{R}$ can be calculated as in the following procedure.

$\operatorname{arcpR}:=\operatorname{proc}(\mathrm{p})$

local eqn, ustar, u:

global beta_erfc, U2, u0:

ustar := 'ustar': u := 'u':

eqn := beta_erfc(ustar) $=\mathrm{p}$ :

fsolve(eqn, \{ustar\}, u0..U2):

assign(\%):

$\mathrm{u}:=$ ustar:

return(u):

end proc:

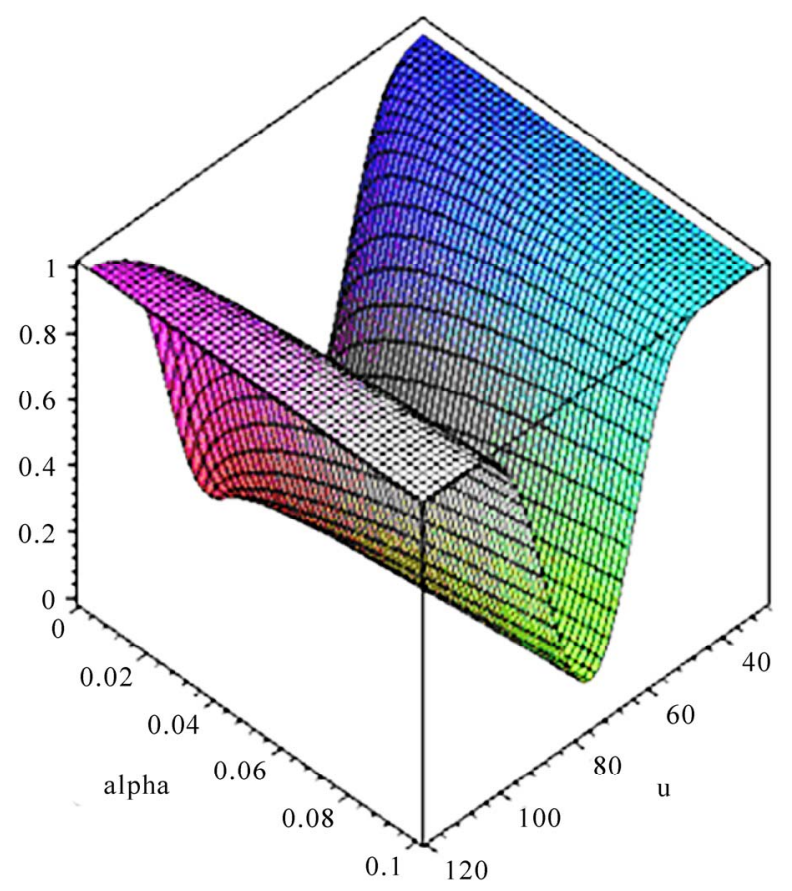

Figure 3. The power surface $1-\beta(u, n)$.

A similar procedure arcpL can be used to calculate $u_{L}$. Of course, only one of the procedures is actually needed due to symmetry. $u_{L}$ and $u_{R}$ satisfy $u_{R}=2 u_{0}-u_{L}$. Note that $u_{0}-u_{L}=u_{R}-u_{0}$ is precisely the amount by which the population mean $u$ must change in one direction or the other in order that the probability of a Type II error does not exceed $p$.

Once the values $u_{L}(p)$ and $u_{R}(p)$ are available, we know that $\beta(u)$ will not exceed $p$ if $u \leq u_{L}$ or $u \geq u_{R}$. Suppose we wish to calculate the probability $p_{*}(p)$ this will happen. Property 8 allows us to do so. As examples, suppose $p=0.2$. Then $\alpha=0.01$ yields $p_{*}=0.04$ while $\alpha=0.05$ yields $p_{*}=0.06$ and $\alpha=$ 0.10 yields $p_{*}=0.09$. In the latter case, we then can say there is a $9 \%$ chance of committing a Type II error that does not exceed $20 \%$, that is to say, $9 \%$ of all possible values for the new population mean yield a Type II error not exceeding $20 \%$.

Although $p_{*}$ is smaller than $\alpha$ for most cases of interest its size is easily explained by the rounded shape of $\beta_{u}(u)$. If $p$ is small enough that $u_{L}$ and $u_{R}$ differ significantly from $u_{0}$, the area of the region under $\beta_{u}(u)$ between $u_{L}$ and $u_{R}$ can be nearly one. Since this area is $1-p_{*}, \quad p_{*}$ tends then to be near zero. Interpreted in another way, the tails whose combined size is $p_{*}$ can be quite small for a rounded distribution such as $\beta_{u}(u) . \quad p_{*}$ serves as a measure of and a reminder that keeping the probability of a Type II error below a prescribed level can be quite a challenge. 


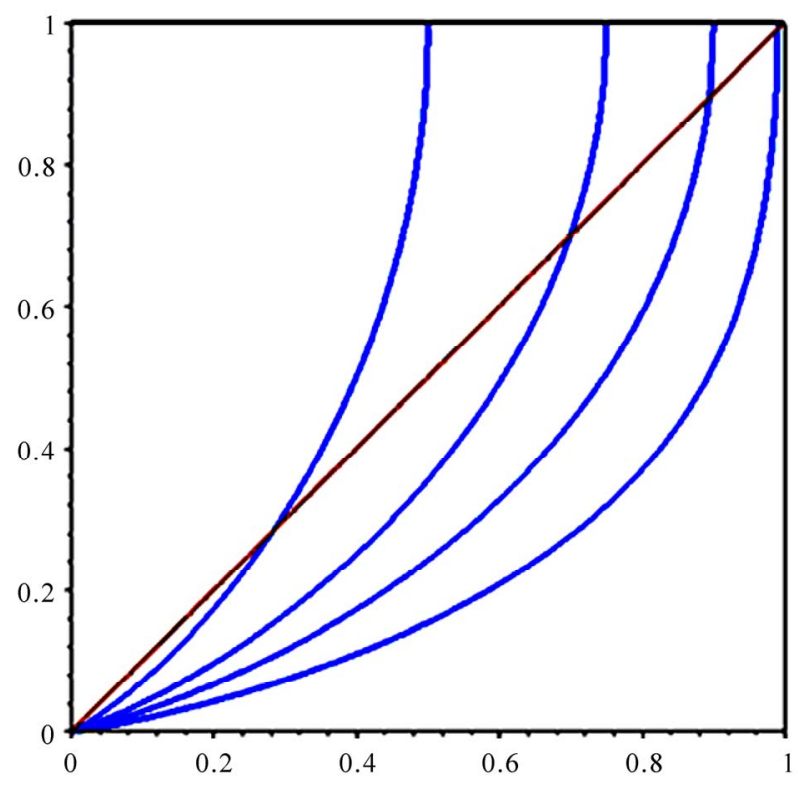

Figure 4. The curves $p_{*}(p)$ for $\alpha=0.01,0.10,0.25,0.5$.

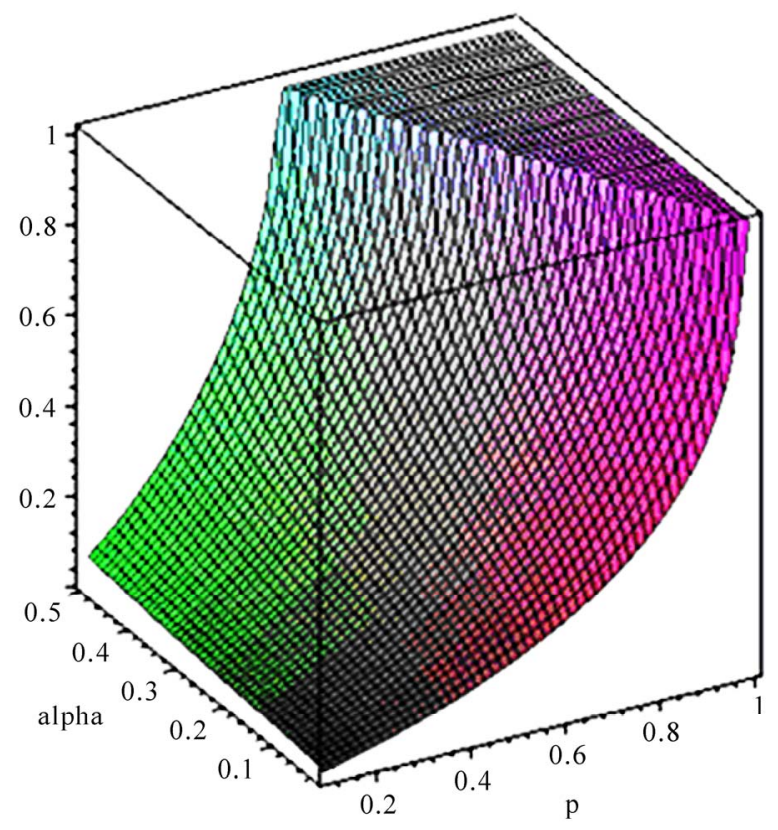

Figure 5. The surface $p_{*}(p, \alpha)$.

The following procedure calculates $p_{*}(p)$. Figure 4 depicts graphs of the functions $p_{*}(p)$ (the blue curves) vs $p$ (the brown curve) for selected significance levels $\alpha=0.01,0.10,0.25$, and 0.50 . The horizontal extent of each blue curve is the interval $[0,1-\alpha]$; the vertical extent is the interval $[0,1]$. Figure 5 depicts a more detailed surface plot of $p_{*}(p, \alpha)$.

betastar $:=\operatorname{proc}(\mathrm{p})$
\# Calculate pstar, the probability that the \# probability of a Type II error will not \# exceed p.

local eqn, ustar, u, uL, pstar: global beta_cdf, beta_erfc, U1, u0, CL: ustar := 'ustar': u := 'u': pstar := 'pstar': $\mathrm{uL}:=$ 'uL': eqn $:=$ beta_erfc(ustar) $=\mathrm{p}$ : fsolve(eqn, \{ustar\}, U1..u0): assign(\%):

$\mathrm{uL}:=$ ustar: pstar := 2*beta_cdf(uL): return(pstar):

end proc:

An interval oriented variant of $p_{*}(p)$ can provide additional information. Given two probability levels $p_{1}$ and $p_{2}$ with $0 \leq p_{1}<p_{2} \leq 1-\alpha$, suppose we wish find the probability that the size of a Type II error will be between these values. In this case, Property 9 allows us to calculate this probability. A procedure for performing the necessary calculations can be found in [3].

\section{Summary}

This paper investigated the probability distribution for Type II errors. Several interesting properties of the distribution were obtained. These properties can be used to obtain the same information as that obtained using other commonly used methods. In addition, the properties allow us to quantify several thorny issues in precise ways. The manner in which this can be done was discussed and illustrated using selected Maple procedures for working with the distribution.

\section{Acknowledgements}

The author is indebted to an anonymous referee and to the AM staff for several suggestions which improved the exposition of this paper significantly.

\section{References}

[1] D. C. Montgomery, "Statistical Process Control," John Wiley \& Sons, New York, 1991.

[2] D. S. Wackerly, W. Mendenhall, and R. L. Scheaffer, "Mathematical Statistics with Applications," Duxbury Press, 1996.

[3] S. Thompson, "Maple Worksheets for Investigating Type II Errors,” 2010.

http://www.radford.edu/thompson/TypeIIErrors/index.ht $\mathrm{ml}$

[4] Maplesoft, Waterloo Maple Inc., Waterloo, 2010. 